\title{
Prevalence of primary and secondary hypertension: studies in a random population sample
}

\author{
GÖRAN BERGLUND, OVE ANDERSSON, LARS WILHELMSEN
}

investigation schedule has been described. ${ }^{6}$ We report here the prevalence of blood pressure increases above $175 \mathrm{~mm} \mathrm{Hg}$ systolic or $115 \mathrm{~mm} \mathrm{Hg}$ diastolic, or both, at screening in men

\section{Summary}

The prevalence of primary and secondary hypertension was determined in a random sample of 7455 Swedish men aged 47 to 54 years. Three hundred and sixty-one men were undergoing treatment for hypertension. Seven hundred and ninety-eight men who had blood pressures above $175 / 115 \mathrm{~mm} \mathrm{Hg}$ at preliminary screening were recalled for further blood pressure measurements. Those on treatment and all the untreated men whose blood pressures were still over $175 / 115 \mathrm{~mm} \mathrm{Hg}$ then underwent extensive investigation for secondary hypertension. Renal parenchymal hypertension was found in $25(3.6 \%)$ patients, renovascular hypertension in four $(0.6 \%)$, and other forms of secondary hypertension in $11(1.6 \%)$. The investigation led to surgical treatment in only two cases $(0.3 \%)$. The low prevalence of secondary hypertension, especially surgically curable forms of hypertension, makes routine screening for these cases unnecessary, at least when patients with hypertension have been found at screening. These data must be taken into account in planning community control programmes in hypertension.

\section{Introduction}

The prevalence of hypertension has been well documented in epidemiological studies in many countries, ${ }^{1-3}$ but the proportion of the total hypertensive population with demonstrable causes of hypertension (renal, vascular, or endocrine disease) is not known. This is probably because relatively extensive investigations are necessary to identify secondary forms of hypertension with reasonable certainty. Since only a small proportion of the hypertensive population probably has secondary hypertension many patients have to be investigated. Furthermore, the extensive tests must be applied to an unselected hypertensive population obtained by screening a population sample, as secondary forms of hypertension are probably over-represented in hospital series.

It is important, however, to establish the prevalence of secondary hypertension since the investigations that are currently undertaken before treatment are largely aimed at detecting secondary forms of hypertension. The design and scope of the investigations should thus directly depend on the number of cases of secondary hypertension expected. A high prevalence of secondary, presumably curable, hypertension would warrant more extensive preliminary investigations.

At the hypertension clinic at our hospital we had a unique opportunity to study a large hypertensive population of middleaged men. ${ }^{4-6}$ The series was obtained by blood-pressure screening of a random population sample. The standardised

\footnotetext{
Medical Department I, Sahlgren's Hospital, University of Göteborg, Göteborg, Sweden

GÖRAN BERGLUND, MD, physician

OVE ANDERSSON, MD, physician

LARS WILHELMSEN, MD, head of department aged 47-54 years and the prevalence of secondary hypertension among these men.

\section{Population and methods}

The men with hypertension were recruited as part of a multifactorial primary preventive trial started in $1970 .^{46}$ They came from the intervention group of that trial, which consisted of a randomly selected third of all men aged 47-54 years (born 1915-22 and 1924-5) resident in Göteborg. Men born in 1923 took part in a separate study. The 9996 men were invited to have their blood pressure measured, and $7455(75 \%)$ attended. Those who were not receiving antihypertensive treatment and whose blood pressures were above $175 \mathrm{~mm} \mathrm{Hg}$ systolic or $115 \mathrm{~mm} \mathrm{Hg}$ diastolic were recalled to have their blood pressure taken again after two weeks. Those whose blood pressures were still higher than $175 / 115 \mathrm{~mm} \mathrm{Hg}$ and all those who were on antihypertensive treatment then underwent investigation for secondary hypertension. ${ }^{6}$

\section{INVESTIGATION}

Investigations included a complete physical examination with auscultation of the heart and lungs, palpation of peripheral pulses, examination of the ocular fundi, and a battery of laboratory tests including measurement of serum electrolytes and creatinine, urine tests for albuminuria and urinary sediment, urine culture, and determination of the renal concentration capacity with a 13-hour thirst test and, if the result was abnormal, a vasopressin tannate test.

The screening methods ${ }^{4}$ and the investigations at the hypertension clinic $^{6}$ have been reported in detail elsewhere and are described only briefly. Blood pressure was measured in the seated position after five minutes' rest. Screening was performed in the afternoon, when blood pressure is at its highest, ${ }^{1}$ and the limits, $175 \mathrm{~mm} \mathrm{Hg}$ systolic and 115 $\mathrm{mm} \mathrm{Hg}$ diastolic, corresponded to values of $160 \mathrm{~mm} \mathrm{Hg}$ and 101 $\mathrm{mm} \mathrm{Hg}$ measured in the morning in the same population. ${ }^{6}$ Serum electrolytes and creatinine were determined using a Technicon AutoAnalyzer, albuminuria with a dip test (Albustix, Ames), and urine sediment and quantitative urine cultures with the routine methods of the hospital. Urine osmolarity, the renal concentration capacity after 13 hours' thirst, was determined according to the method of Hood, ${ }^{7}$ and if the result was below $700 \mathrm{mmol}$ (mosmol)/ $\mathrm{kg} \mathrm{H}_{2} \mathrm{Oa}$ vasopressin tannate test was performed. ${ }^{8}$ Isotope renography was carried out in the 287 men born in 1915, 1916, 1920, and 1921 using the standard method and apparatus (Nucab, Sweden). Intravenous pyelography (in 52 men) and renal aortography (in 12) were also performed using standard methods. Catecholamine concentrations were determined in all 58 men born in 1921 using a method described by von Euler and Floding. ${ }^{9}$

CRITERIA OF SECONDARY HYPERTENSION

On the basis of the results of these investigations and a thorough examination of each patient's case notes a diagnosis was recorded using the criteria given below.

Renoparenchymal disease - (1) The diagnosis of chronic glomerulonephritis required persistent or intermittent albuminuria or haematuria, or both, without there being another cause of these abnormalities. (2) Renal tuberculosis was recorded when there was a history of pulmonary or renal tuberculosis, or both, with tubercle bacilli in the urine. (3) Gouty nephropathy was recorded in the presence of known arthritis urica and lowered concentration ability (vasopressin tannate test $<800 \mathrm{mmol} / \mathrm{kg} \mathrm{H}_{2} \mathrm{O}$ ). (4) Renal dysplasia was defined as one small 
kidney without a history of urinary tract infections. A kidney was considered small if it was over $2 \mathrm{~cm}$ shorter than the other kidney and less than $10 \mathrm{~cm}$ in length with oddly-shaped or club-like calices on the intravenous pyelogram. ${ }^{10}$ (5) Phenacetin nephropathy was recorded in patients with a long-standing history of abuse, lowered urinary concentration ability, and signs of papillary necrosis on the intravenous pyelogram. (6) Chronic pyelonephritis required a history of recurrent urinary tract infections and a lowered urinary concentration ability.

Renovascular disease-Screening for renovascular disease was carried out by isotope renography. Patients with abnormal renograms were admitted to hospital for renal aortography and other investigations. Arteriographic verification was required for a diagnosis of renal artery stenosis or intrarenal occlusion. Abnormally high ipsilateral renin secretion or normalisation of the blood pressure after surgery was not required for this diagnosis. Isotope renography was also performed if there were clinical signs of renovascular disease-for example, abdominal murmurs, drug resistant or accelerating hypertention, or increasing serum creatinine levels.

Primary aldosteronism-A hospital-verified diagnosis was required. Patients with spontaneous hypokalaemia or hypokalaemia during diuretic treatment whose serum potassium levels did not become normal after treatment were admitted to hospital for investigation.

Primary hyperparathyroidism-A hospital-verified diagnosis was required. Serum calcium and phosphorous were not routinely screened.

Phaeochromocytoma-A hospital-verified diagnosis was needed. Catecholamines in urine were determined only in men born in 1921 .

\section{Results}

\section{PREVALENCE OF HIGH BLOOD PRESSURE}

Of the 7455 men who were screened $1159\left(16^{\circ}\right)$ had blood pressures above $175 \mathrm{~mm} \mathrm{Hg}$ systolic or $115 \mathrm{~mm} \mathrm{Hg}$ diastolic, or both, or were receiving antihypertensive treatment. Six hundred and twenty-two men $\left(54^{\circ}{ }_{0}\right)$ had never been treated and $176\left(15^{\circ}{ }_{0}\right)$ had received antihypertensive treatment before but were not receiving it at the time of screening. Thus 798 men $\left(69^{\circ}{ }_{0}\right)$ were untreated and $361\left(31_{0}^{\circ}\right)$ were on antihypertensive drugs. The treatment in $196\left(54^{\circ}\right)$ of the treated patients seemed to be inadequate because they had blood pressures above $175 \mathrm{~mm} \mathrm{Hg}$ systolic or $115 \mathrm{~mm} \mathrm{Hg}$ diastolic. Only $14^{\circ}$ o (165) of the 1159 men considered to have hypertension were adequately controlled on treatment.

The 798 men who were not on antihypertensive treatment were recalled for further blood pressure measurement after two weeks. Three hundred and twenty-four had blood pressures below the limits at the second measurement and were given appointments for repeat measurements one year later. A further 21 declined further investigation, seven had died or moved from the city, and 30 did not attend for the second blood pressure measurement. The remaining 416 men had blood pressures over $175 \mathrm{~mm} \mathrm{Hg}$ systolic or 115 diastolic at the second reading and were invited to attend for further investigation.

Of the 361 patients who were already receiving treatment 24 declined further treatment, nine had died or moved, 36 had appointments for check-ups with another doctor, and 19 did not attend for further investigation. The remaining 273 patients joined the 416 untreated patients for further investigation.

\section{PREVALENCE OF SECONDARY HYPERTENSION}

Of the 689 patients investigated at the hypertension clinic $\left(9^{\circ}{ }_{0}\right.$ of the total population sample) 25 were found to have renoparenchymal disease. Fifteen patients had chronic glomerulonephritis, which had been previously confirmed in all cases (by a positive finding on renal biopsy or proteinuria over $2 \mathrm{~g}$ /day). Persistent haematuria alone was present in three cases, but in all three patients intrarenal kidney stones were judged to be the cause of the haematuria. Previously known renal tuberculosis was present in four cases and gouty nephropathy in three cases (one of which was previously known). There were two cases of renal dysplasia. One was already known and the other was detected by isotope renography. Previously known phenacetin nepropathy was present in one patient.

Renovascular hypertension was found in four patients, two of whom had main artery stenosis (one unilateral and one bilateral). The other two had intrarenal vascular occlusion. Three cases were discovered by our investigations and one was known. In addition, there was one known case of aortic coarctation. one of primary aldosteronism, and two of primary hyperparathyroidism. The patient with aldosteronism and the two with hyperparathyroidism had undergone surgery.
In seven cases of hydronephrosis (two known and five newly detected) we could not determine whether the hypertension was caused by the hydronephrosis and the resulting partial obstruction to flow. Three patients did not become normotensive after operation and needed continuing antihypertensive treatment, and four patients were not operated on because they became normotensive with drug treatment. No case of phaeochromocytoma was discovered.

Thus a specific cause of hypertension was shown or reasonably suspected in 40 of the 689 patients $(6 \%$ ). Eight patients (two with renal tuberculosis, one with renal dysplasia, the patient with primary aldosteronism, the two patients with primary hyperparathyroidism, and two of the seven patients with hydronephrosis) had previously undergone surgical treatment. In only two cases (one of renal artery stenosis and one of unilateral hydronephrosis) did the investigations lead to surgical intervention. Both these patients required antihypertensive treatment 12 months after their operations, but lower doses were needed than before.

\section{Discussion}

Men were considered to have hypertension warranting diagnostic investigation if they had two blood pressure readings above $175 / 115 \mathrm{~mm} \mathrm{Hg}$ or were receiving antihypertensive treatment. The 689 men investigated probably represented a random sample of people with hypertension defined as above and who would have been expected to participate in a screening investigation of this kind. The 2546 men who failed to participate in the screening programme had a mortality rate three times higher than normal within three years and were registered with the Temperance Board more often than patients who took part but there was no higher incidence of diseases associated with hypertension. ${ }^{11}$ The arbitrarily chosen blood pressure limits for hypertension may seem high, but they were chosen on the basis of the findings in a study that showed a high incidence of organic disease attributable to hypertension above these limits. ${ }^{3}$ Several factors in the screening procedure may explain why the blood pressure distribution was displaced to the right - that is, towards higher values. These factors have already been discussed in detail. ${ }^{6}$

The methods of recognising secondary hypertension were those normally used in clinical practice. Owing to the limited capacity of the laboratory a renogram was carried out in only four randomly selected age groups. In patients with hypertension refractory to treatment investigation for renovascular causes was also carried out in the other age groups, and most cases of renovascular hypertension in which surgical treatment was possible were therefore probably detected. Parenchymatous kidney disease without abnormal laboratory findings and with normal serum creatinine levels and concentration capacity may have been present without being detected. These patients probably had minor kidney damage, however, which in most cases would not have been treated other than with hypotensive drugs even if discovered. Primary aldosteronism was probably excluded with a relatively high degree of certainty. Primary hyperparathyroidism as a cause of hypertension may also have been present in more than the two cases that were known since serum calcium was not measured routinely.

This study has resulted in two important findings. Firstly, most of the hypertensive population were unaware of their hypertension and had had no treatment. In those who were being treated blood pressure control was unsatisfactory in most cases. These data agree closely with results from other population studies. ${ }^{12}$ Experience from different centres indicates that vigorous measures must be adopted to control the problem of hypertension. About $80 \%$ of the population studied had consulted a doctor during the previous three years. ${ }^{13}$ If doctors could be persuaded to record the blood pressure of all patients irrespective of the cause of the visit and to investigate and treat those found to have raised blood pressures expensive population-based screening would be unnecessary. Educating doctors about hypertension and establishing guidelines for effective treatment and follow-up should constitute the first measures in a programme for controlling hypertension in the community. 
Such measures will probably produce results only in the long term, however. Population-based screening may therefore be a realistic alternative at present and is likely to have a more rapid impact on the problem of hypertension.

Secondly, the prevalence of secondary hypertension was low $\left(5 \cdot 8^{\circ} \mathrm{o}\right)$ and that of surgically curable cases ever lower. The prevalence of secondary hypertension in this study was lower than other estimates. ${ }^{14-17}$ Our analysis is, however, the first one to be done in subjects derived from screening a total population. Furthermore, we studied only men aged 47-54 years. The prevalence of secondary hypertension might be higher in women or in younger men. Our results suggest, however, that in middleaged men extensive investigations aimed at detecting secondary hypertension are not necessary in those found to have hypertension at screening. In patients with hypertension referred to hospitals secondary hypertension is probably over-represented and more extensive routine investigations might be justified. Renography as a screening instrument for renovascular hypertension cannot be recommended. The prevalence of renovascular hypertension was low, and there were many false-positive renograms. ${ }^{18}$ Our results support the findings of recent costbenefit analyses of urography and renography as screening instruments for renovascular hypertension ${ }^{19}$ and of comparisons of surgical and medical treatment of renovascular hypertension. ${ }^{20}$ The tests we used led to surgery for two patients, neither of whom was cured. Both our results and those quoted above thus suggest that in planning for community control of hypertension secondary hypertension should not be sought with advanced investigative methods. Instead, only patients whose history, physical status, or routine test results suggest secondary hypertension should be submitted to further investigation. The remainder, more than $95 \%$, should be given drug treatment.

Whether or not the standard investigations we performed in all men with hypertension are also unnecessary is more difficult to assess. Electrocardiography, chest $x$-ray examination, the tests for albuminuria, and measurements of serum creatinine may show hypertensive organ damage in a relatively high percentage of patients. ${ }^{21} 22$ The importance of identifying those with a poorer prognosis, thus enabling more intensive treatment and follow-up, justifies retaining these procedures in the standard investigations of the hypertensive patient.

The study was supported by grants from the Swedish Medical Research Council, B-74-19X-4229-01A-03C, from the Swedish Association against Heart and Chest Diseases, and from the Bank of Sweden Tercentenary Fund. We wish to thank Dr Michael McCall, Perth, Australia, for valuable suggestions.

\section{References}

${ }^{1}$ National Health Survey: Hypertension and Hypertensive Heart Disease in Adults. Washington, Public Health Service Publications, 1966.

2 Böe, J, Humerfelt, S, and Edervang, F, Acta Medica Scandinavica, 1957, suppl 321.

3 Tibblin, G, Acta Medica Scandinavica, 1967, suppl 470.

4 Wilhelmsen, $L$ Tibblin, G, and Werkö, L Preventive Medicine, 1972 $1,153$.

${ }^{5}$ Berglund, G, and Wilhelmsen, L, Acta Medica Scandinavica, 1975, 198, 291.

6 Wilhelmsen, L, Berglund, G, and Werkö, L, Preventive Medicine, 1973, 2, 57 .

${ }^{7}$ Hood, B, et al, Nordisk Medicin, 1965, 74, 1111.

8 Wardener, H E de, The Kidney. An Outline of Normal and Abnormal Structure and Function. Churchill, London, 1958.

9 von Euler, U S, and Floding, I, Scandinavian fournal of Clinical and Laboratory Investigation, 1956, 8, 288.

${ }^{10}$ Hood, B, et al, Scandinavian fournal of Urology and Nephrology, 1968, 2, 95.

11 Wilhelmsen, L, Ljungberg, S, and Werkö, L, fournal of Chronic Diseases, in press.

12 Hypertension Study Group, Circulation, 1972, 444, 263.

13 Wilhelmsen, L, Hur Finna Populationens Hypertoniker? I Hypertoni 74, ed G Berglund and L Werkö. Mölndal, Lindgren and Söner, 1975.

14 Dustan, H P, in The Heart and Circulation-Second National Conference on Cardiovascular Diseases. Research I, p 334. Washington DC, Federation of American Societies for Experimental Biology, 1965.

15 Gifford, R W, Milbank Memorial Fund Q, 1969, 47, 170.

${ }^{16}$ Laragh, J H, American fournal of Medicine, 1972, 52, 565.

17 Ferguson, R K, Annals of Internal Medicine, 1975, 82, 761.

18 Berglund, G, Drugs, 1976, 11, Suppl No 1, p 60.

${ }^{9}$ McNeil, B J, et al, New England fournal of Medicine, 1975, 293, 216.

${ }^{20} \mathrm{McNeil}, \mathrm{B} \mathrm{J}$, and Adelstein, S J, New England fournal of Medicine, 1975, 293, 221.

21 Wickstrand, J, et al, British Heart fournal, in press.

22 Berglund, G, Aurell, M, and Wilhelmsen, L, Acta Medica Scandinavica, $1976,199,25$.

\title{
Randomised controlled trial of antibiotics in patients with cough and purulent sputum
}

\author{
NIGEL C H STOTT, ROBERT R WEST
}

vaginal infections, gastrointestinal upsets, and otitis media over the next six months were the same in both groups, but fewer new upper respiratory infections were experienced by the doxycycline-treated patients.

There is no consensus among doctors about using

Two hundred and twelve adults with cough and purulent sputum of up to one week's duration were allocated randomly to treatment with doxycycline or placebo capsules for up to 10 days. Cough, purulent sputum, feeling "off colour," and time off work lasted as long in treatment and control groups, but running nose persisted for a shorter time in the doxycycline group. The number of new episodes of lower respiratory tract infections,

Welsh National School of Medicine, Heath Park, Cardiff CF4 4XN NIGEL C H STOTT, MRCP, senior lecturer, general practice unit ROBERT R WEST, PHD, lecturer, department of community medicine

antibiotics in patients with cough and purulent sputum, and these results indicate that otherwise healthy people with these symptoms will usually get better without antibiotic treatment.

\section{Introduction}

Respiratory infections are so common and sometimes so trivial that they are not always regarded as diseases. There is also no clear relation between many clinical respiratory syndromes and the organisms isolated, because a range of symptoms and signs is caused by the same organisms and vice versa. ${ }^{1}$ Host resistance is equally variable: dietary, socioeconomic, hereditary, 